Supporting Information

\title{
A Trojan-Horse Strategy by In-Situ Piggybacking onto Endogenous Albumin for Tumor-Specific Neutralization of Oncogenic MicroRNA
}

Gijung Kwak, Hyosuk Kim, Jooho Park, Eun Hye Kim, Hochung Jang, Geonhee Han, Sun Young Wang,

Yoosoo Yang, Ick Chan Kwon* and Sun Hwa Kim* 


\section{EXPERIMENTAL SECTION \\ MALDI-TOF MS analysis}

The MALDI-TOF spectra of AM-Anti-21and MI-Anti-21 was run on a Kratos AXIMA CFR (Shimadzu Biotech, Kyoto Japan), Mass Spectrometer in positive ion mode and using linear mode. The data were analyzed using software Kompact v.2.4.1 provided by the instrument.

\section{Dynamic light scattering}

The size distribution of Albumin-Anti-21 was examined by dynamic light scattering (DLS, Zetasizer Nano, Malvern Instruments, UK). Disposable cuvettes (\#DTS0012, Malvern Instruments) were used, and the measurement angle was $173^{\circ}$ Backscatter. For zeta potential measurement, disposable folded capillary cell (\#DTS1070, Malvern Instruments) was used. The data were analyzed using software Zetasizer software v.7.13 provided by the instrument.

\section{Endosomal escape of MSA-Anti-21}

MSA-Anti-21 was prepared with Cy5.5-labeled MI-Anti-21. U87 cells were plated in $\Phi 35$ glass-bottom dishes at a density of $3 \times 105$ cells per dish with $2 \mathrm{~mL}$ culture medium and incubated for $24 \mathrm{~h}$. The cells were incubated with saline, Anti-21, or MSA-Anti-21 equivalent to $300 \mathrm{nM}$ Anti-21 in serum-free medium. After $8 \mathrm{~h}$, the cells were incubated with $50 \mathrm{nM}$ LysoTrackerTM Green DND-26 in a fresh medium for $30 \mathrm{~min}$. The live cells were counterstained with DAPI and observed with a confocal laser microscope (FV1000, Olympus, Japan). 


\section{SUPPORTING FIGURES}

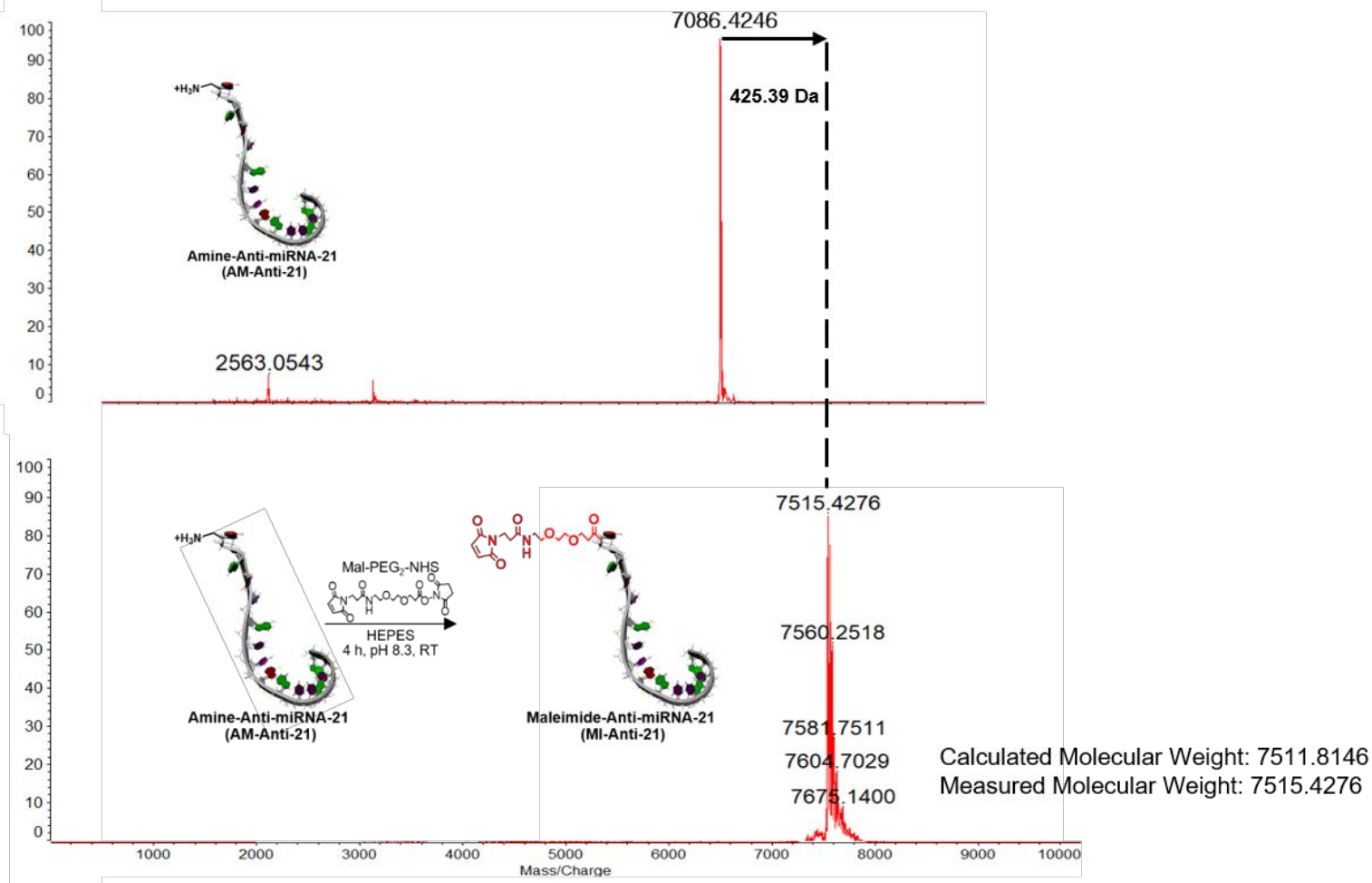

Supporting Figure 1. MALDI-TOF spectra of amine-modified anti-miRNA-21 (AM-Anti-21) functionalized with Mal-PEG $2-\mathrm{NHS}_{2}$ ester. 
a

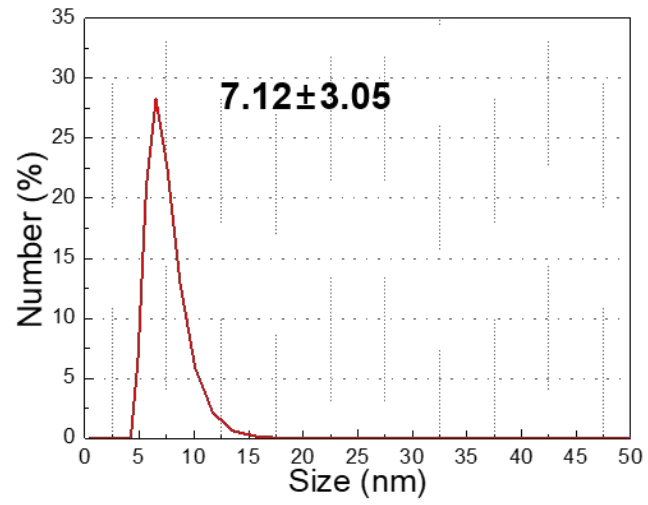

b

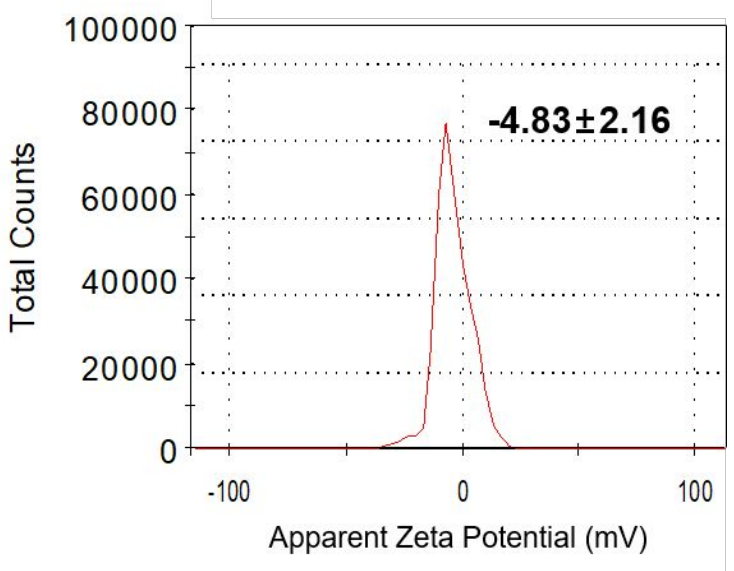

Supporting Figure 2. Characterization of Albumin-Anti-21 by (a) particle size measurement (b) zeta potential measurement. 
a

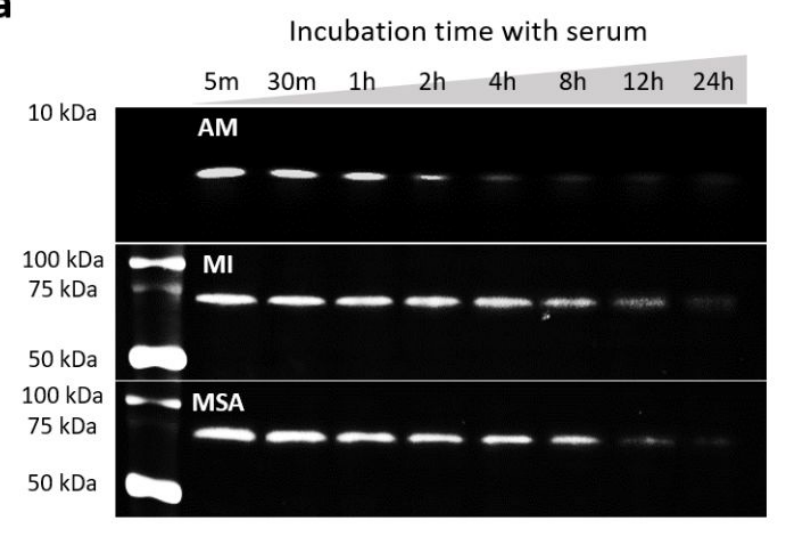

b

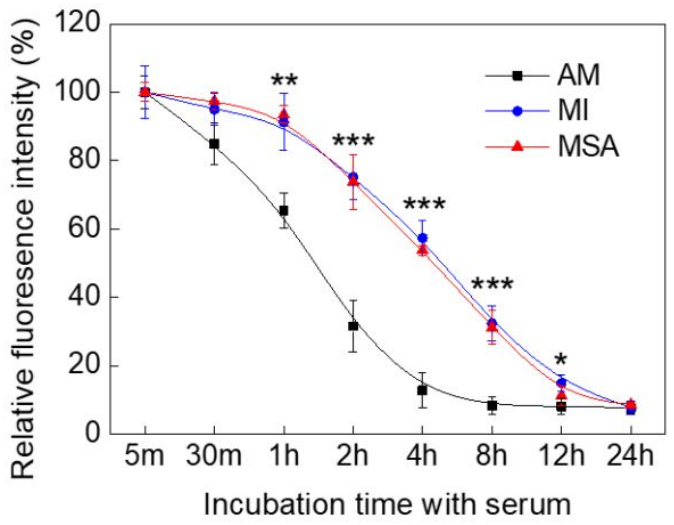

Supporting Figure 3. (a) Representative fluorescence image by electrophoresis of serum incubated in vitro with anti-21 modified with amine (AM), maleimide (MI) and MSA for various time up to $24 \mathrm{~h}$. (b) Quantitative presentation of electrophoresis. $\mathrm{n}=3$ separate experiment, mean \pm s.d. $*: p<0.05, * * *: p<0.001$ vs. AM group by Dunnett's multiple comparisons test. 


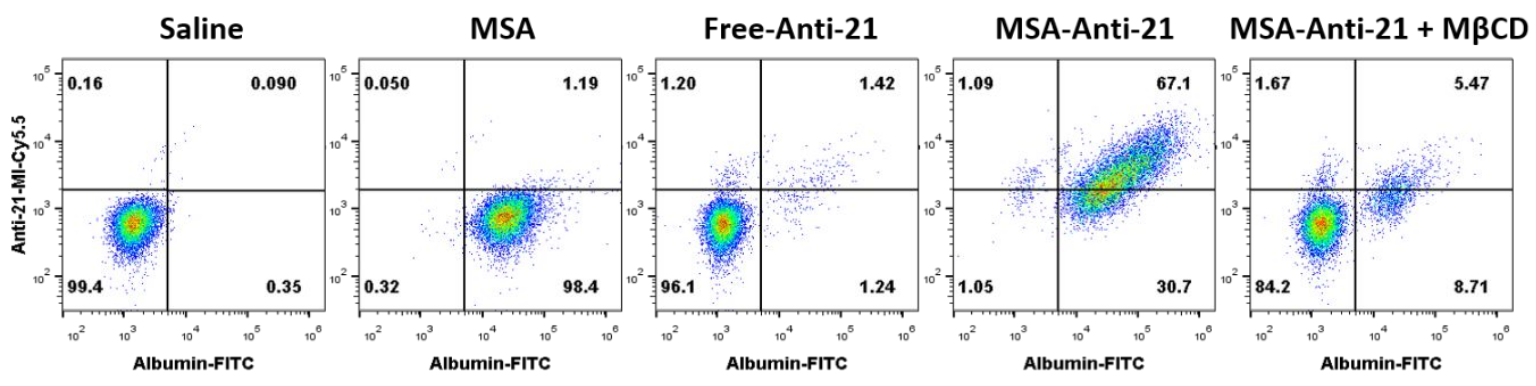

Supporting Figure 4. Uptake of albumin, free Anti-21, Albumin-Anti-21 and Albumin-Anti21 plus the endocytosis inhibitor, $\mathrm{M} \beta \mathrm{CD}$, by U87 cells, observed by flow chemistry. 

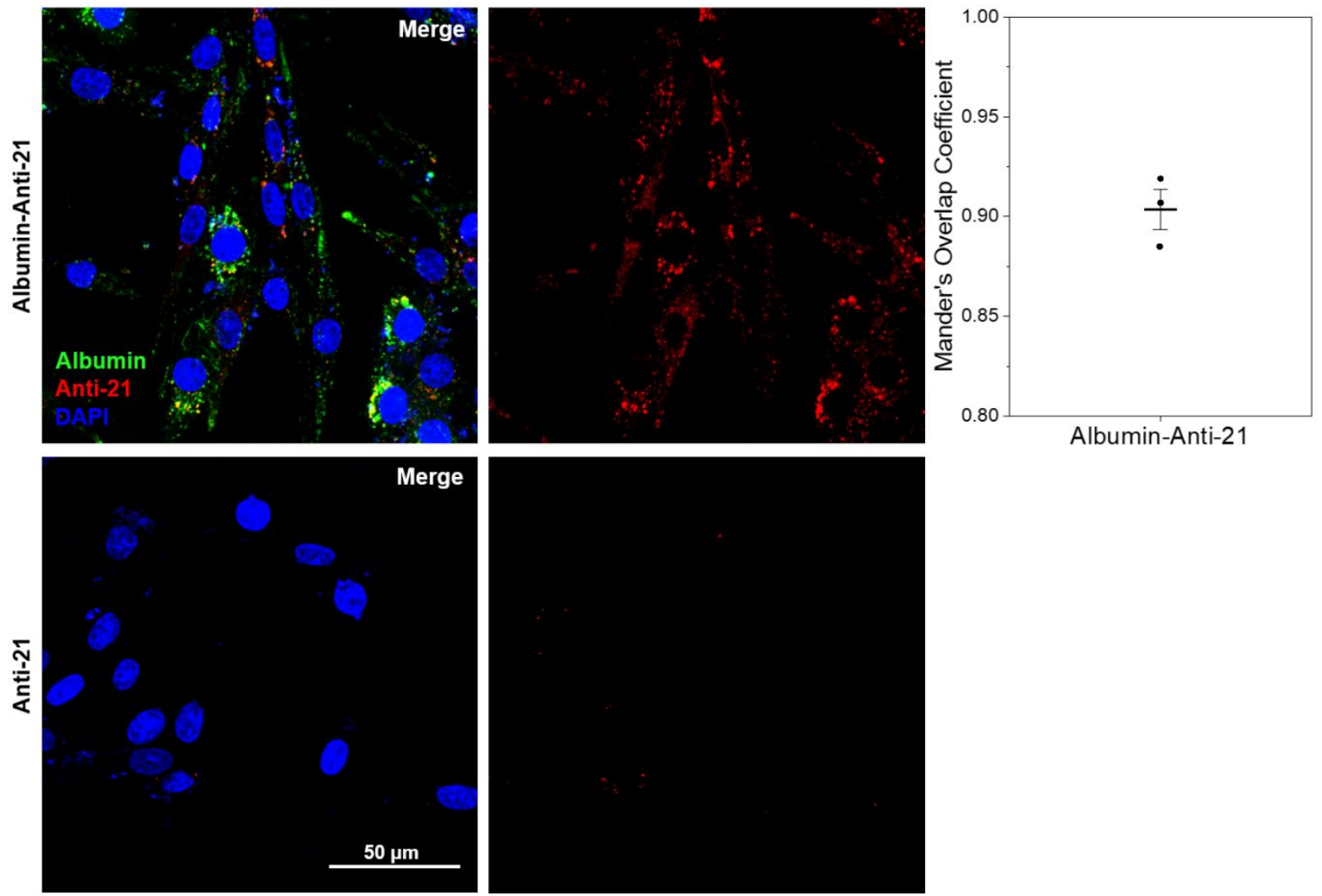

Supporting Figure 5. Confocal microscopy image and Mander's Overlap Coefficient for Albumin-Anti-21 (300 nM) uptake by U87 cells. 
a

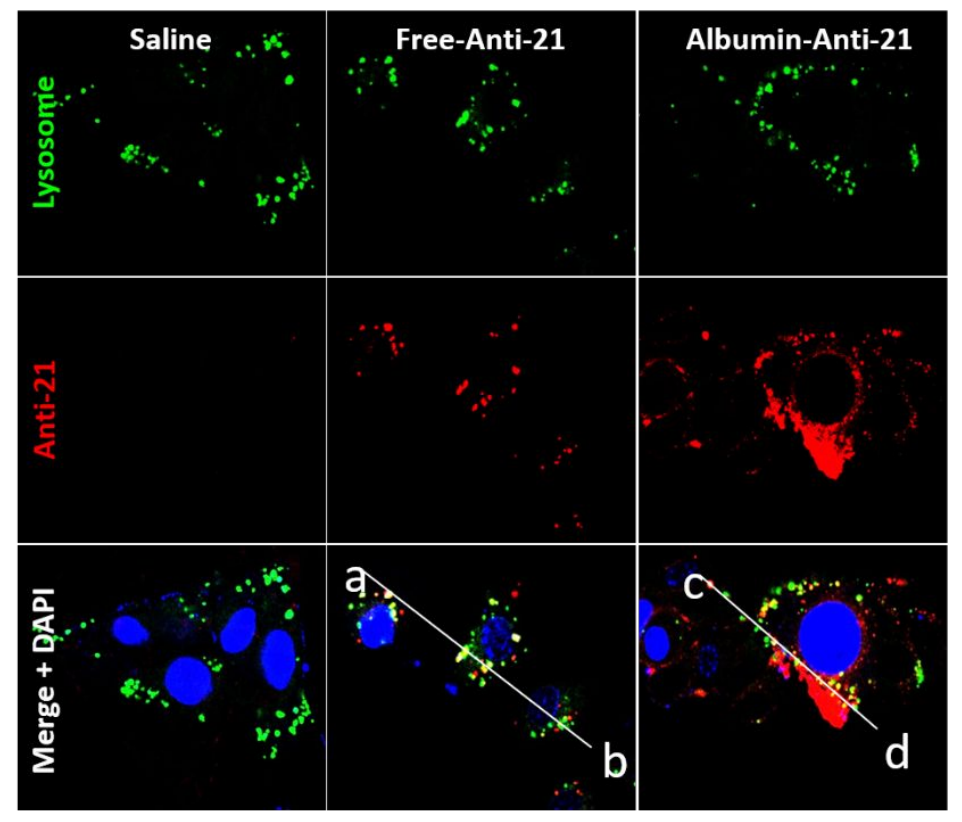

b
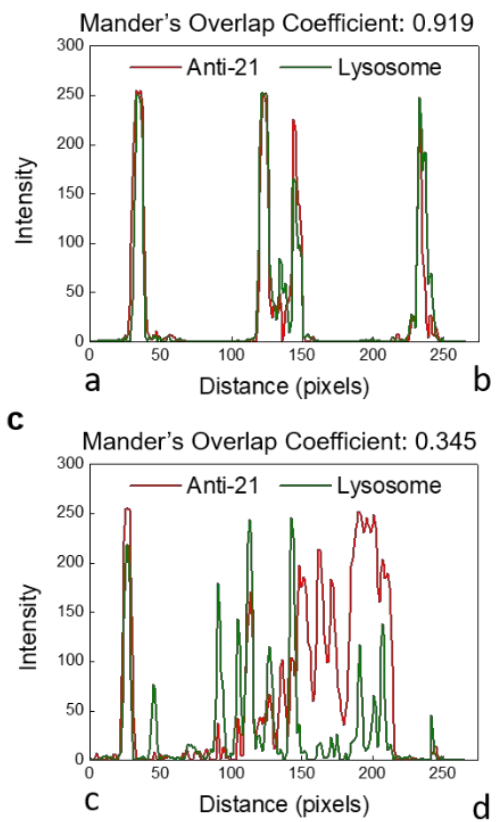

Supporting Figure 6. (a) Endosomal escape of free Anti-21 and Albumin-Anti-21 in U87 cells, observed by confocal microscopy. (b, c) Fluorescence intensities of Anti-21 and lysosomes at the a-b line (b) and c-d line (c) in U87 cells treated with free Anti-21 or Albumin-Anti-21 indicated in panel a. 


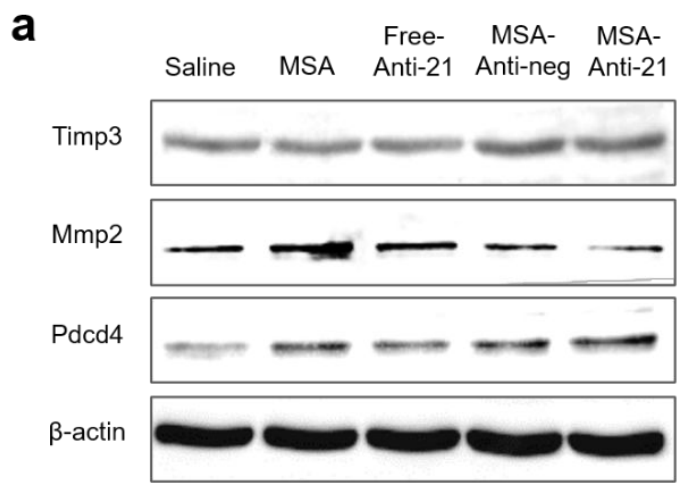

C
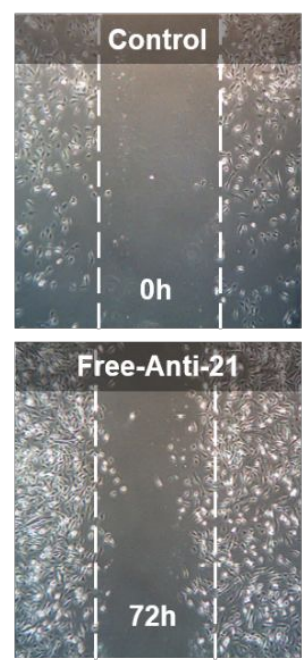
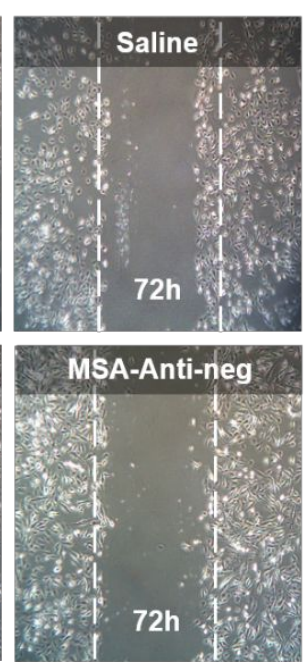

b

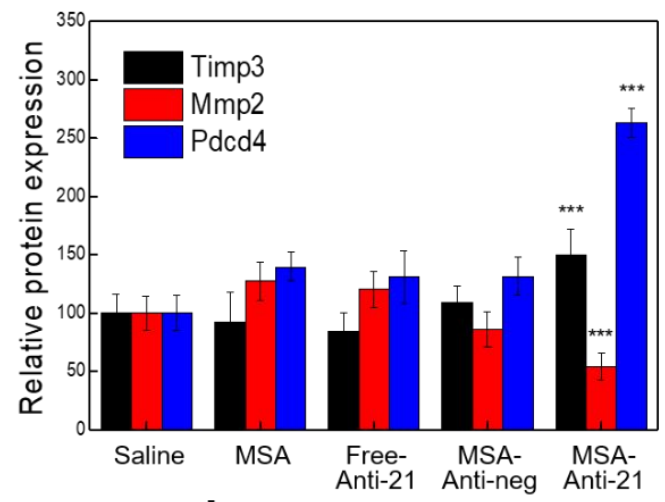

d
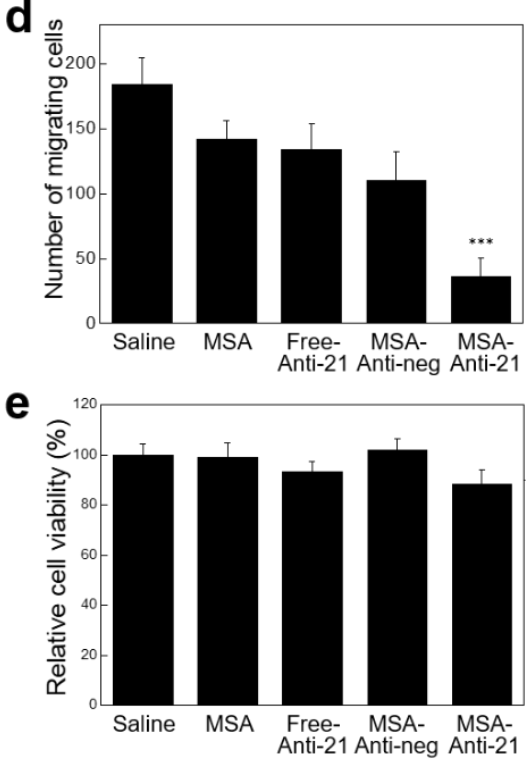

Supporting Figure 7. (a) Representative Western blot images demonstrating changes in Timp3, Mmp2 and Pcdc4 expression in U373 MG cells induced by MSA, free Anti-21, MSAAnti-neg or MSA-Anti-21. (b) Quantitative presentation of Western blot shown in Figure 5a ( $\mathrm{n}=3$ separate experiments). (c) Representative images of scratch-wound healing assays demonstrating the migration of U373 MG cells $72 \mathrm{~h}$ after treatment with MSA, free Anti-21, MSA-Anti-neg or MSA-Anti-21. (d) Quantification of migrating cells in supporting figure 3c ( $\mathrm{n}=3$ separate experiments). (e) Viability of U373 MG cells treated with MSA, free Anti-21, MSA-Anti-neg or MSA-Anti-21 ( $\mathrm{n}=6$ separate experiments). Data are presented as means \pm s.d. (***: $p<0.001 v s$. saline group; Dunnett's multiple comparisons test). 


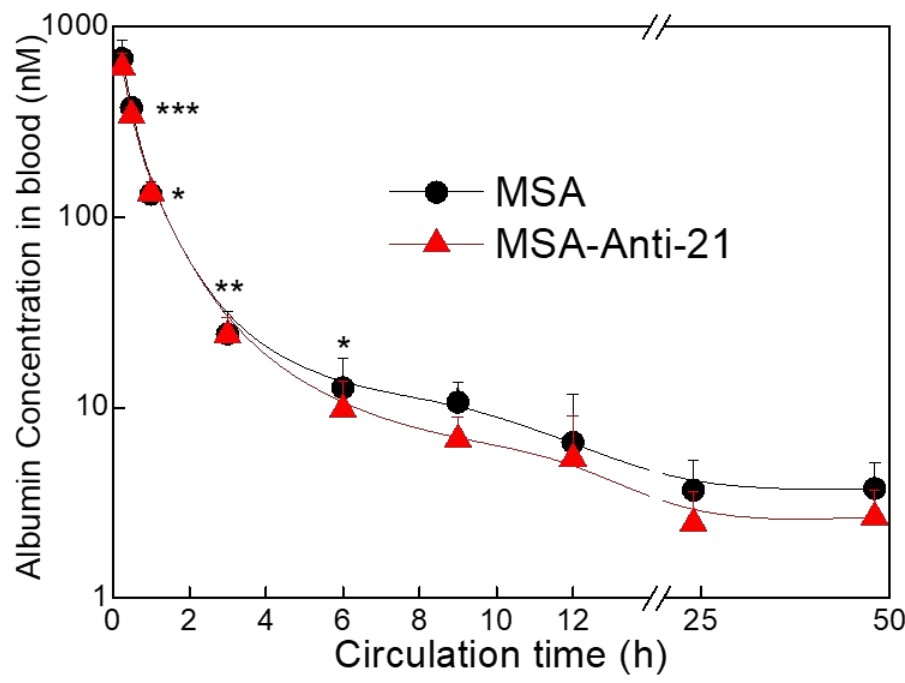

Supporting Figure 8. Time profiles of albumin concentration in blood (logarithmic scale) for MSA-Anti-21 and MSA groups ( $\mathrm{n}=3$ per group). MSA group was labeled with MI-Cy5.5, and MSA-Anti-21 group was labeled with Anti-miR-21-Cy5.5. 


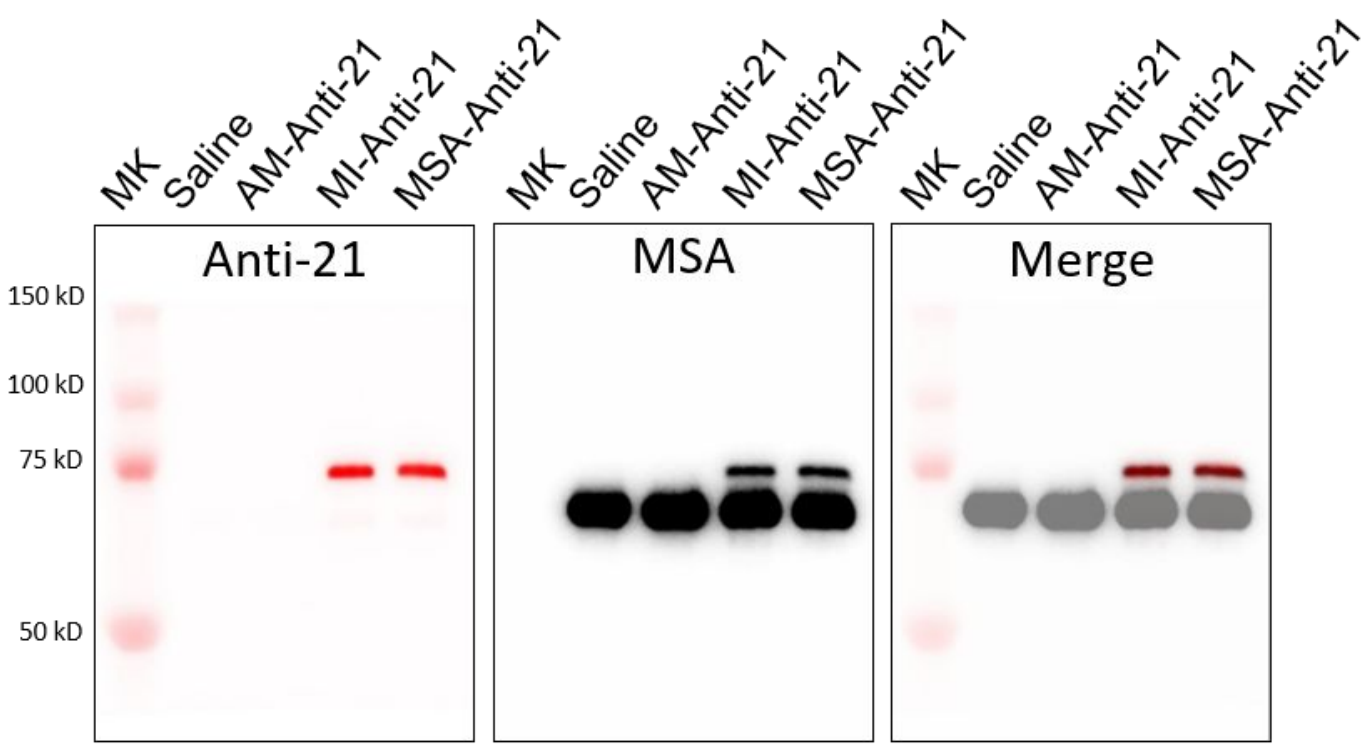

Supporting Figure 9. Representative Western blot images of plasma $1 \mathrm{~h}$ after administration of saline, AM-Anti-21, MI-Anti-21 or MSA-Anti-21 in BALB/c mice. 


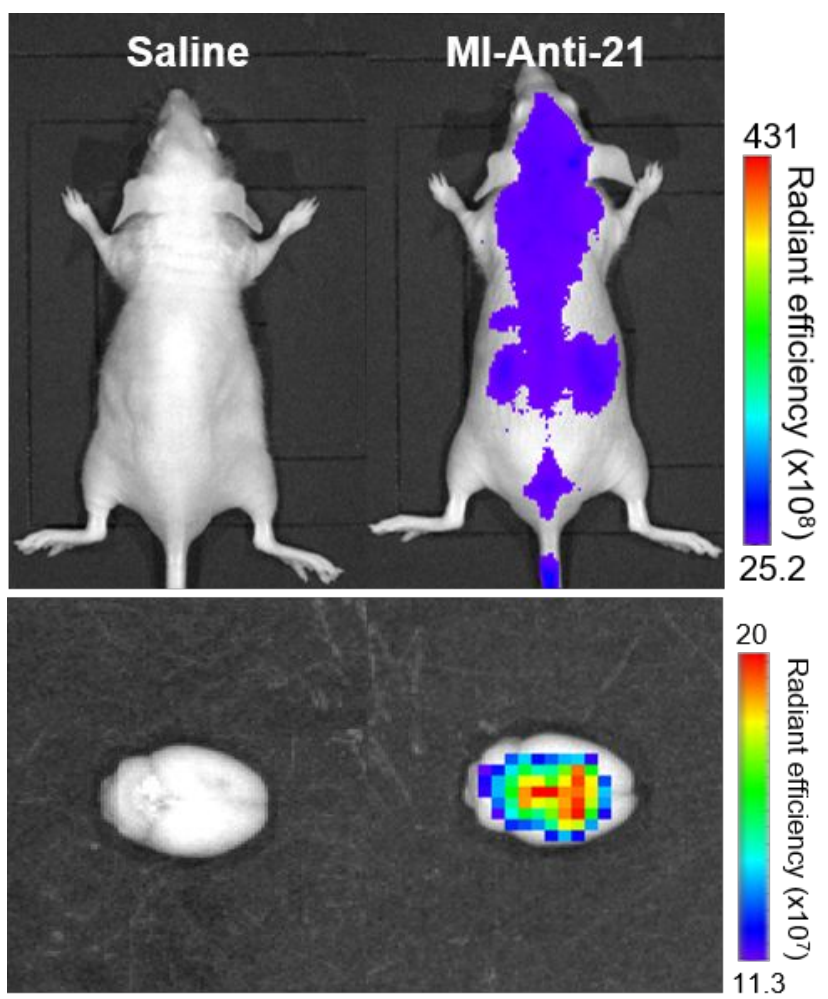

Supporting Figure 10. In vivo images showing the whole-body distribution of MI-Anti-21 in an orthotopic mouse model of U87 and ex vivo images of the brain extracted at $3 \mathrm{~h}$. 


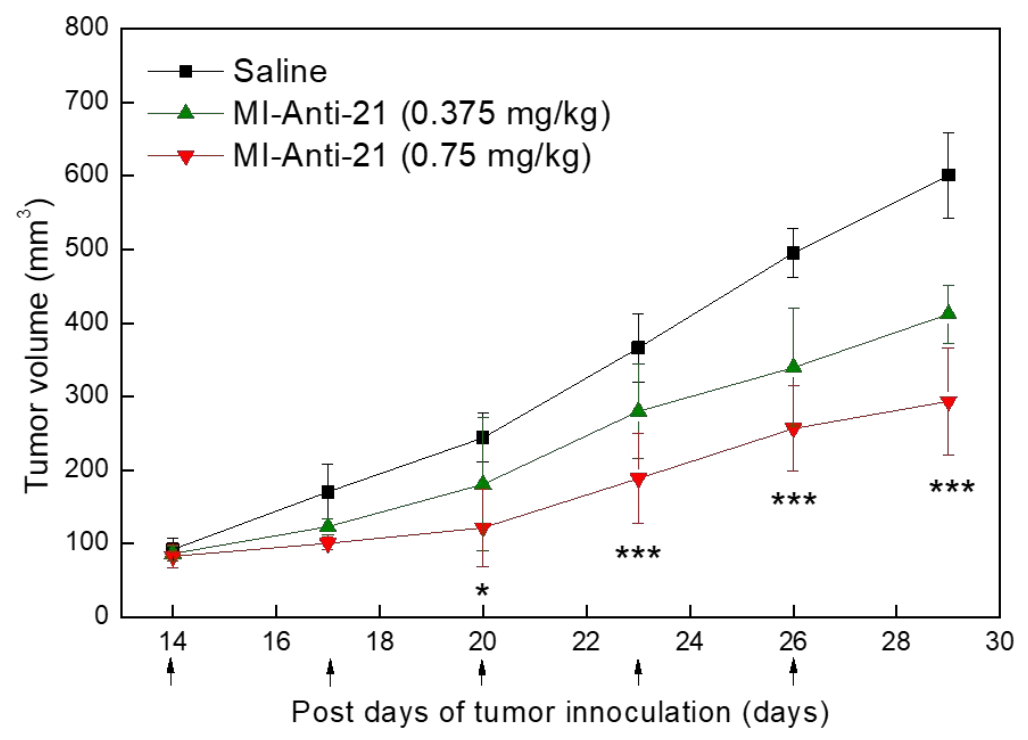

Supporting Figure 11. Changes in tumor volume in BALB/c-nude mice treated with saline, MI-Anti-21 $(0.375 \mathrm{mg} / \mathrm{kg})$ or MI-Anti-21 $(0.75 \mathrm{mg} / \mathrm{kg})(\mathrm{n}=6$ per group). Arrows on the Xaxis indicate administration times with 3-days interval. 
a

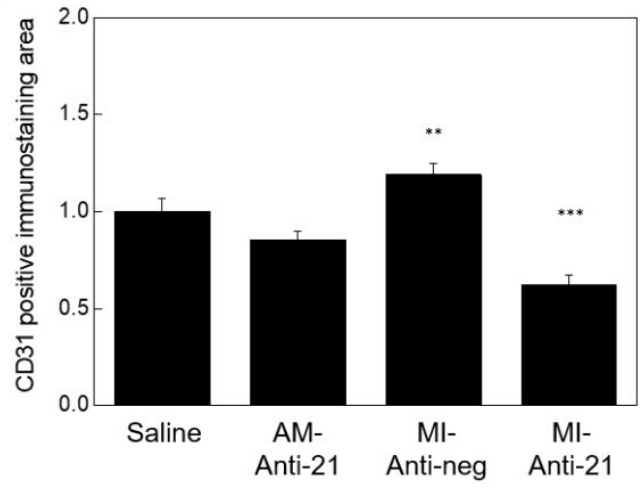

b

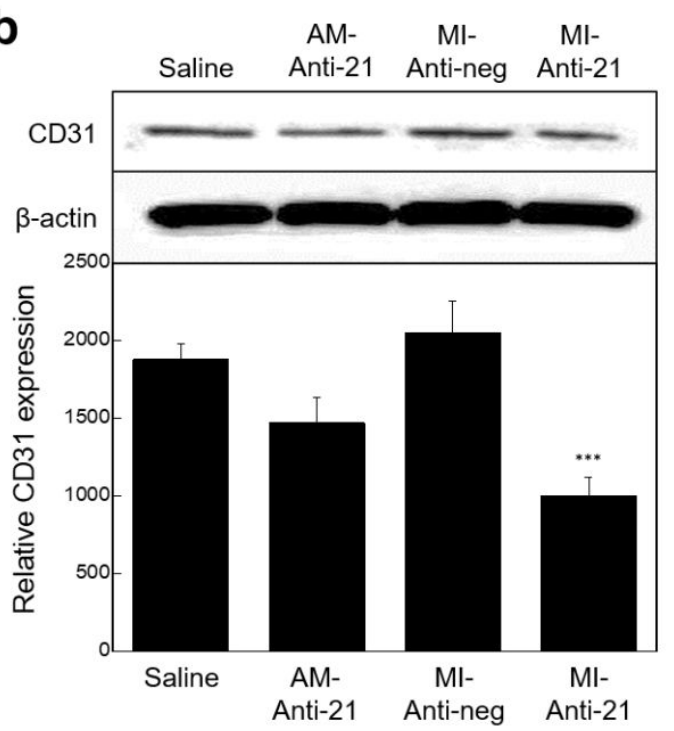

Supporting Figure 12. (a) Quantification of CD31 expression on tumor sections ( $\mathrm{n}=3$ per group). (b) Representative western blot images and quantitative data showing changes in the expression of CD31 in U87 tumor tissues ( $n=3$ per group). 
a
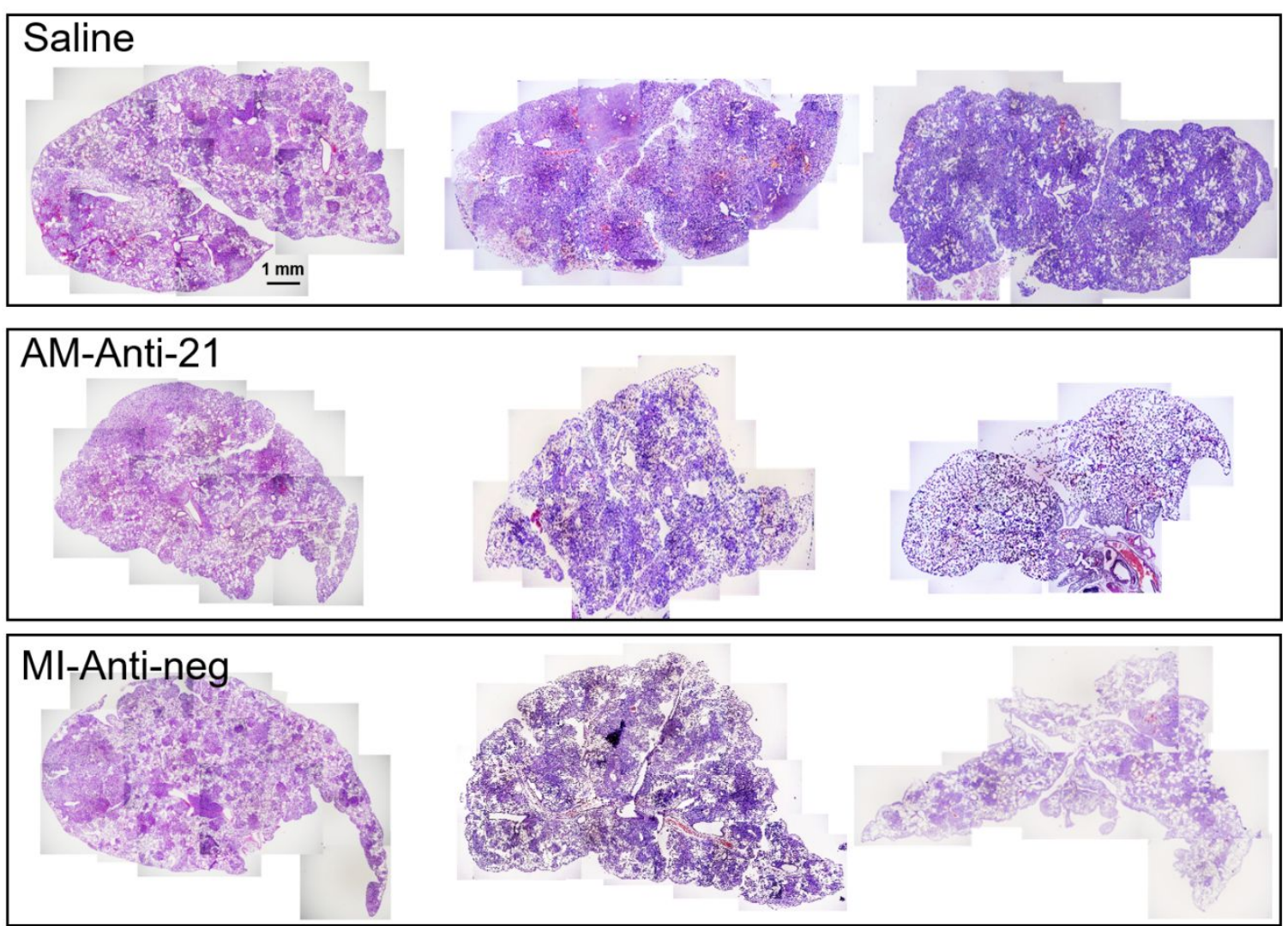

MI-Anti-21
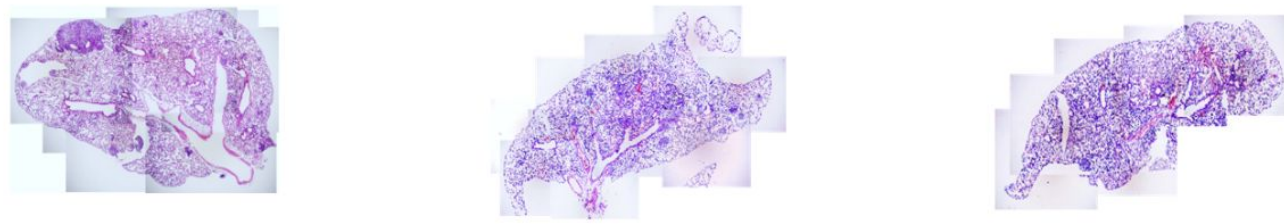

b

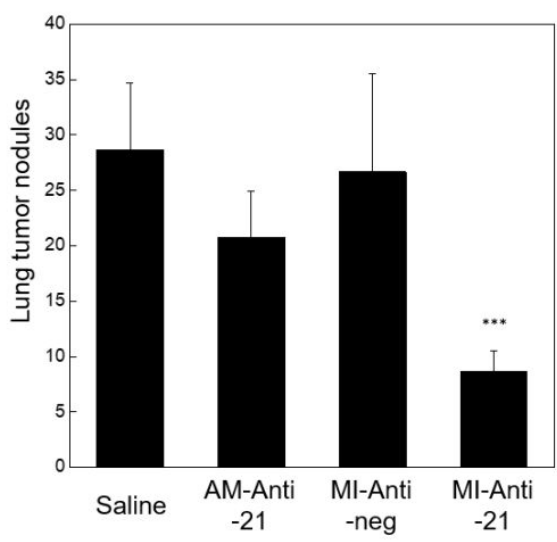

Supporting Figure 13. (a) Images of H\&E-stained sections of whole lungs extracted from the mouse tumor metastasis models treated with saline, AM-Anti-21, MI-Anti-neg or MI-Anti-21. (b) Quantification of the number of lung metastatic nodules ( $n=3$ per group). 

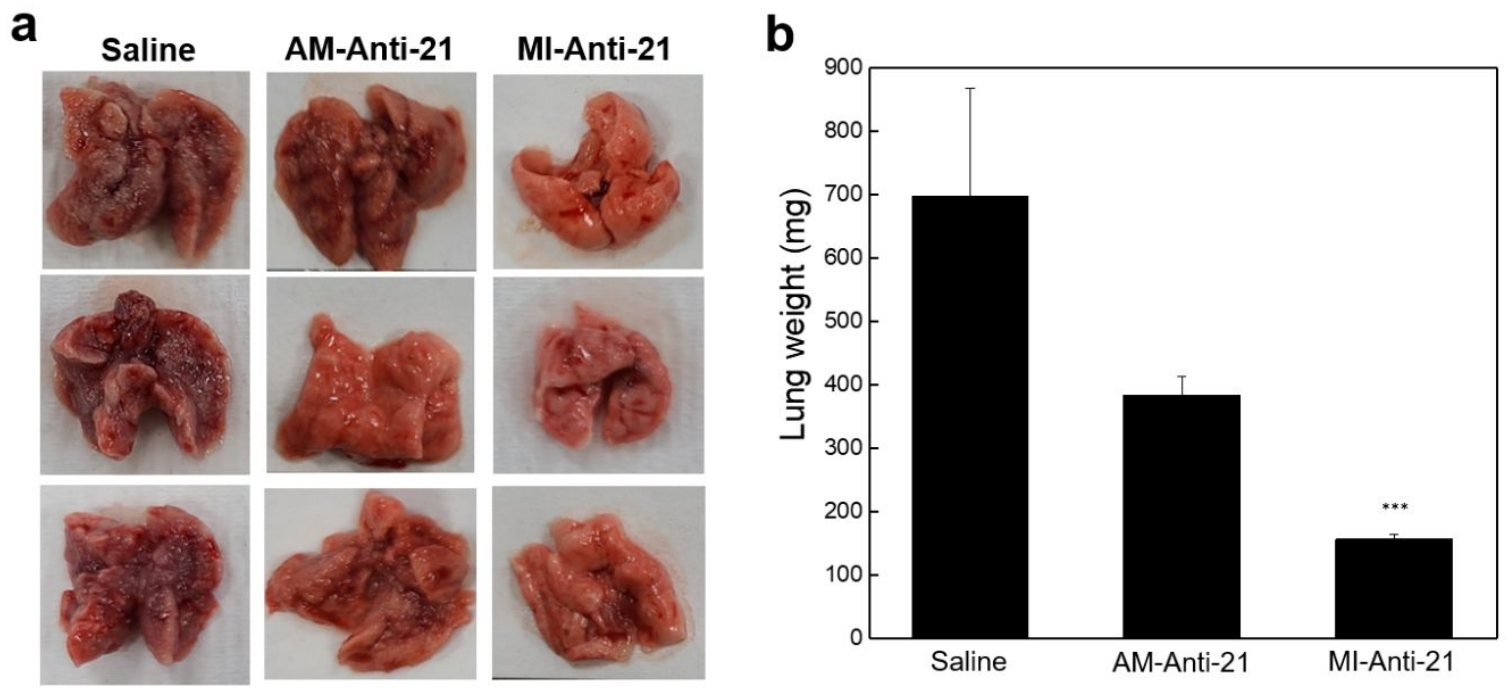

Supporting Figure 14. (a) Images and (B) average weight of whole lungs extracted from the mouse tumor metastasis models treated with saline, AM-Anti-21, or MI-Anti-21. 


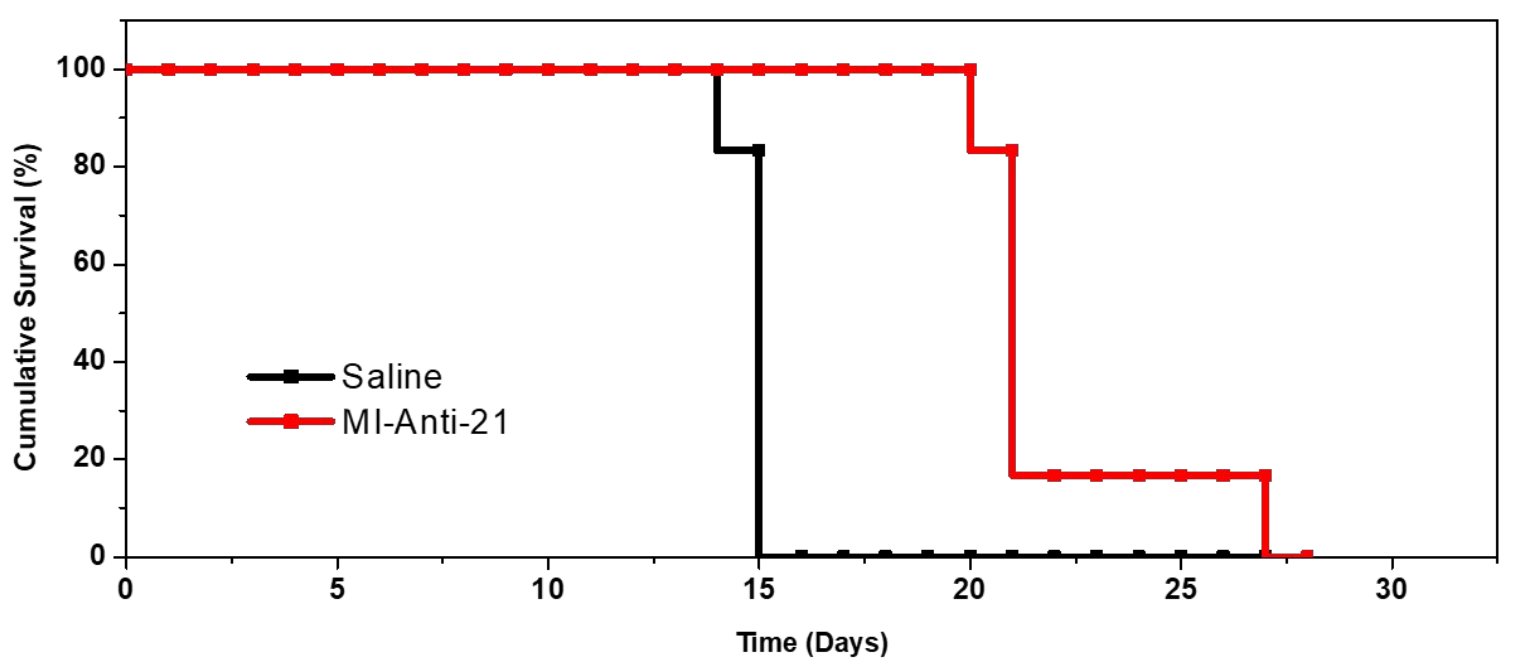

Supporting Figure 15. Survival curve of U87 lung metastasis mouse model. Cultured U87 cells were inoculated intravenously into Balb/c nude mice on day 0 . After tumor cell injection, animals received intravenous injections of saline, or MI-Anti-21 equivalent dose of $0.75 \mathrm{mg} / \mathrm{kg}$ of anti-miRNA via the tail vein. The treatment was repeated 3 more times with a 3-day interval. ( $n=6$ per group). 


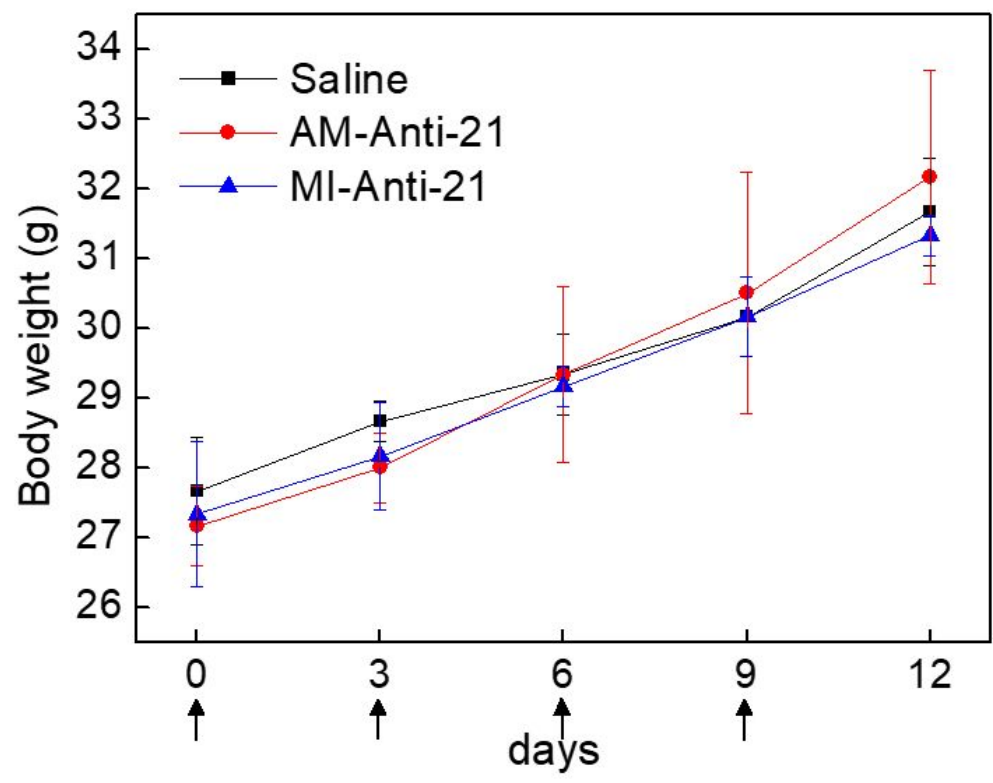

Supporting Figure 16. Systemic toxicity tests showing changes in body weight in BALB/c mice treated with saline, AM-Anti-21, or MI-Anti-21 ( $n=3$ per group). Arrows on the $\mathrm{X}$-axis indicate administration times with 3-days interval. 